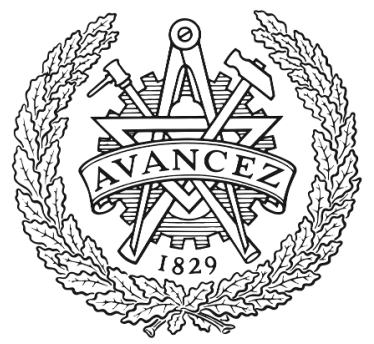

CHALMERS

UNIVERSITY OF TECHNOLOGY

\title{
MonteCoffee: A programmable kinetic Monte Carlo framework
}

Downloaded from: https://research.chalmers.se, 2023-04-26 11:33 UTC

Citation for the original published paper (version of record):

Jørgensen, M., Grönbeck, H. (2018). MonteCoffee: A programmable kinetic Monte Carlo framework. Journal of Chemical Physics, 149(11). http://dx.doi.org/10.1063/1.5046635

N.B. When citing this work, cite the original published paper. 


\title{
MonteCoffee: A programmable kinetic Monte Carlo framework
}

\author{
Mikkel Jørgensen a) and Henrik Grönbeck ${ }^{\text {b) }}$ \\ Department of Physics and Competence Centre for Catalysis, Chalmers University of Technology, \\ 41296 Göteborg, Sweden
}

(Received 29 June 2018; accepted 31 August 2018; published online 18 September 2018)

\begin{abstract}
Kinetic Monte Carlo (kMC) is an essential tool in heterogeneous catalysis enabling the understanding of dominant reaction mechanisms and kinetic bottlenecks. Here we present MonteCoffee, which is a general-purpose object-oriented and programmable kMC application written in python. We outline the implementation and provide examples on how to perform simulations of reactions on surfaces and nanoparticles and how to simulate sorption isotherms in zeolites. By permitting flexible and fast code development, MonteCoffee is a valuable alternative to previous kMC implementations. Published by AIP Publishing. https://doi.org/10.1063/1.5046635
\end{abstract}

\section{INTRODUCTION}

Understanding catalytic reaction kinetics is an essential component to develop the field of heterogeneous catalysis. Reaction kinetics can be evaluated through kinetic measurements yielding a macroscopic view of the reaction, or through computer-aided simulations that serve to understand microscopic reaction mechanisms. In particular, methods based on electronic structure calculations have become increasingly important over the past decades. ${ }^{1-3}$ A general approach is to link electronic structure calculations with mean-field kinetics, which allows for understanding the average kinetic behavior of uniform catalysts, where the kinetics can be formulated in terms of coverages. However, for systems with different types of sites, such as nanoparticles, there is a need to follow the reaction in greater detail. A direct way of achieving this is by solving Newton's equations of motion, as is achieved by Molecular Dynamic (MD) simulations. However, MD simulations are of limited use in reaction kinetics as the time steps are on the order of vibrational frequencies, which renders chemical reactions rare events. For example, in $\mathrm{CO}$ oxidation to $\mathrm{CO}_{2}$, an adsorbed $\mathrm{CO}$ molecule makes about $10^{11}$ vibrations per $\mathrm{CO}_{2}$ formation step. To overcome this time scale separation issue, kinetic Monte $\mathrm{Carlo}^{4,5}$ (kMC) is often used to simulate reaction kinetics. In $\mathrm{kMC}$, a number of reactive events are defined, and their occurrences are simulated by random number generation. Thus, kMC simulations coarse-grain phase-space to exclude vibrations and solely focus on chemical transitions.

In the field of heterogeneous catalysis, several kMC implementations use the lattice kMC method, ${ }^{6-11}$ which represents the system by a lattice of sites. One challenge in lattice $\mathrm{kMC}$ is to model dynamic site-changes, such as redox reactions, which has been tackled using multi-lattice approaches. ${ }^{2,6}$ Moreover, while a lattice-based approach provides a simple representation of the sites, the method is cumbersome

a)Electronic mail: mikjorge@chalmers.se

b)Electronic mail: ghj@chalmers.se to apply to complex geometries such as nanoparticles. As an alternative to lattice $\mathrm{kMC}$, graph-theoretical codes have been developed, ${ }^{11,12}$ where a global neighbor list is used to represent the site connectivity. This permits simulations of complex geometries. In the present implementation, the focus is on code flexibility, which is desirable as each system where $\mathrm{kMC}$ is applied often is unique. We present a simple and fully flexible framework that allows for rapid kMC code development.

MonteCoffee is an open-source object-oriented programmable (OOP) application, ${ }^{13}$ which has the advantage of quick code development and extensibility. The code is written in python as this is a popular high-level programming language, which is malleable and easy to comprehend. Additionally, python has the advantage that it can be combined with other programmable applications such as the Atomistic Simulation Environment ${ }^{14}$ (ASE). With an object-oriented approach, a number of challenging tasks are straightforward, such as advanced evaluation of adsorbate-adsorbate interactions, dynamic system variations, and descriptor-based energy landscapes. The code uses neighbor-lists to represent the site connectivity, which is more flexible than the lattice kMC method. Using neighbor-lists is similar to the graph-theoretical approaches; however, in MonteCoffee, the user directly controls the site-connectivity. The code implements the firstreaction method (FRM) algorithm ${ }^{15}$ and other algorithms can straightforwardly be implemented.

In this paper, the basic structure and functionality of the MonteCoffee framework is outlined. The main focus is the code implementation and possibilities for extension. The capability of the code is demonstrated in two different examples, namely, $\mathrm{CO}$ oxidation over a nanoparticle with a descriptor-based energy landscape and adsorption isotherms in a zeolite.

\section{KINETIC MONTE CARLO}

kMC simulations typically discretize phase-space into a set of energy basins, which are separated by chemical transitions. This avoids simulating vibrations and focuses on 
the chemical reactions. ${ }^{15}$ Thus, in kMC simulations, a system is defined as a set of sites where a number of reactive events can proceed. Event execution is simulated in time, based on random number generation. This achieves the basic purpose of kMC simulations, to solve the chemical master equation

$$
\frac{\mathrm{d} P_{\alpha}}{\mathrm{d} t}=\sum_{\beta} W_{\alpha \beta} P_{\beta}-W_{\beta \alpha} P_{\alpha},
$$

where $P_{\alpha}$ is the probability for the system being in state $\alpha$ and $W_{\alpha \beta}$ is the transition rate from state $\beta$ to $\alpha$. Here, a state is defined by a specific occupation of the sites. From the generated states and transitions, data such as turnover frequencies (TOFs) and coverages can be extracted and the detailed reaction trajectory can be followed.

The MonteCoffee software implements the First Reaction Method $^{15}$ (FRM). FRM is similar to the Variable Step Size Method (VSSM), also called the n-fold way. ${ }^{15}$ FRM executes events chronologically based on randomly generated times of occurrence,

$$
t_{\alpha \beta}=t-\frac{\ln u}{W_{\alpha \beta}},
$$

where $t_{\alpha \beta}$ is the time the event occurs, $t$ is the current time, and $u$ is a random uniform number in $[0,1[$.

The FRM algorithm consists of three main parts, which are illustrated in Fig. 1:

1. Initialize simulation (T,p), generate reaction times, set time $=\mathbf{0}$ : This part sets the reaction conditions and initializes the system, sites, coverages, and lists used for bookkeeping. The lists for bookkeeping can include

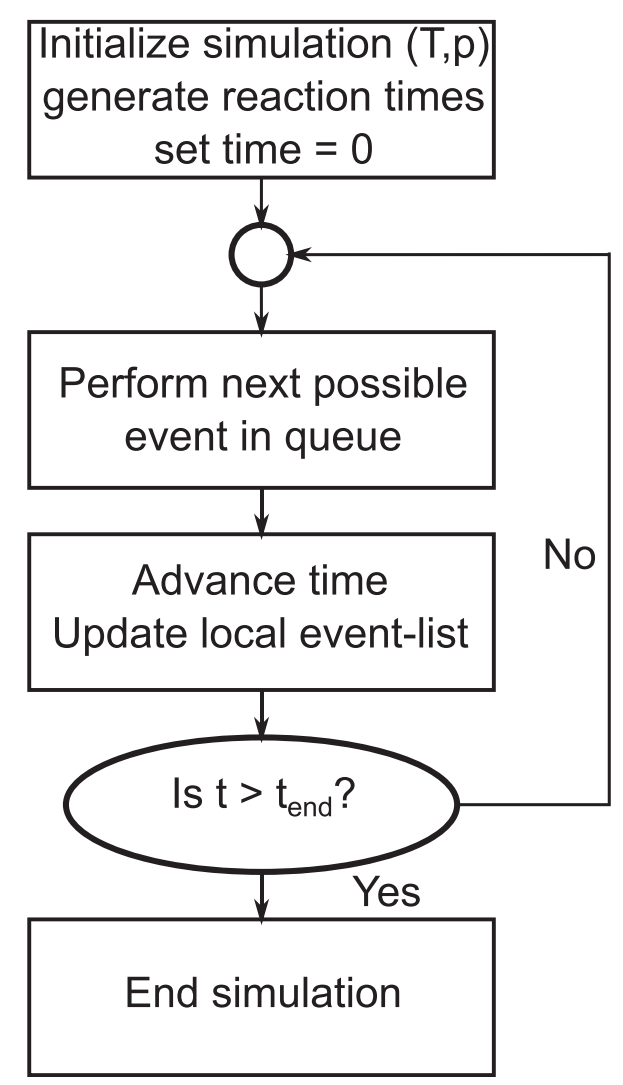

FIG. 1. The first reaction method algorithm. coverages, time steps, the sites where events occurs, etc. Moreover, the initial times of event occurrences are generated, and the simulation time is set to 0 .

2. Perform next event in queue: Performs the chronologically next event according to Eq. (2).

3. Advance time. Update local event-list: The simulation time is updated to the occurrence time of the performed event. Performing the event modifies the lattice locally and nearby events are affected. To enhance performance, the event-list is only updated locally. If nearest-neighbor adsorbate-adsorbate interactions are used, the sites are updated two nearest neighbor distances away from the site where the event was performed.

The loop continues until the simulation has reached its endtime $t_{\text {end }}$. During a simulation, the lists that track statistics grow considerably. Thus, memory is freed at regular intervals by writing these lists to disc as python pickle-files.

kMC simulations are often challenging due to major differences in reaction rates, where slow events are only observed after accelerating the simulation. A rigorous acceleration scheme slowing down individual quasi-equilibrated elementary steps was presented by Chatterjee and Voter ${ }^{16}$ and a proof justifying this method was given in Ref. 17. The task of accelerating simulations is relatively simply achieved in MonteCoffee as it uses custom functions to get rateconstants and perform the events. Two acceleration schemes are available in the code: Slowing down fast events by raising energy barriers manually and the generalized temporal acceleration scheme of Dybeck et al. ${ }^{18}$ The generalized temporal acceleration scheme involves artificially slowing down quasiequilibrated reaction channels to increase the time step of the simulations.

\section{OBJECT-ORIENTED PROGRAMMING}

The current implementation uses object-oriented programming (OOP), which is programming centered around objects and classes. A class is a data-structure that contains variables and functions and objects are specific instances of a class. For example, one can define a Particle class and instantiate a specific particle object by calling oct ahedron $=$ Particle $($ symbol='Pt', $a 0=4.00)$. In this case, an octahedral Pt nanoparticle object is instantiated with a lattice constant of $4 \AA$. After instantiating an object, its variables can be altered during code execution, e.g., as octahedron. symbol = 'Au', which changes the element from $\mathrm{Pt}$ to $\mathrm{Au}$. Classes can also contain functions that are called to change the object. For example, a function set_a0 (a_new) can be defined for the specific particle to change the lattice constant to 3.98 as octahedron.set_a0 (3.98).

In MonteCoffee, class inheritance is used, which means that a base-class passes on some variables and functions to derived classes. In the present implementation, inheritance is used in a manner where base classes are templates for the derived classes. This gives a good overview of the tasks involved in defining a simulation. For example, all events defined by the user are derived from a template-class named EventBase, which ensures that all user-defined events has 
a function that calculates the rate-constants. Thus, if the userdefined derived event class does not contain such a function, the code will give an error message.

It is out of scope to discuss OOP in further detail as it is an extensive subject, with different implementations depending on the programming language. The interested reader is referred to the python documentation ${ }^{19}$ for further information about the use of OOP in python. In Secs. IV-VI, classes are named beginning with an uppercase letter (e.g., Site), whereas objects start with a lowercase letter (e.g., site).

\section{CLASSES AND FUNCTIONS}

Figure 2 shows a general outline of the code modules. The Monte Carlo engine is in the NeighborKMC module, which communicates with a set of different modules, and handles settings as well as simulation logging. The NeighborKMC module relies on a list of events and a system that is composed of a set of sites.

The MonteCoffee software provides classes and functions to define and structure kMC simulations. To adapt the code to a specific problem, classes are defined by the user using inheritance. Figure 3 shows the main classes, essential variables, and functions in the code. This list of variables is restricted to those that are relevant to the present discussion.

\section{A. NeighborKMC class}

The NeighborKMC class performs the simulations and is responsible for the main functionality of the code. It contains a number of variables: parameters is a python-dictionary containing keys and values for temperatures, pressures, and other parameters, which are passed on to the events. All keyvalue pairs in parameters are written in the beginning of the simulation $\log$ and it can conveniently be used to name or comment a simulation. options is a list of settings loaded from a separate file, which contains the frequency of data saving, filenames, etc. time and tend are floats describing the current simulation time and the simulation end-time, respectively. A central object for the NeighborKMC class is the system, which is the system instance that the simulation is modeling. events is the list of reactive events, and frm_times is the list of generated times of occurrence for the events. events is populated with Event objects in the order they are defined. basin_vars represent a set of variables used to steer the generalized temporal acceleration scheme. ${ }^{18}$

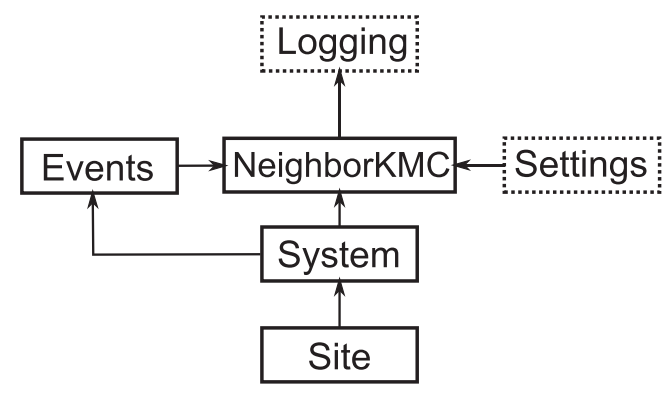

FIG. 2. The main modules and their relationships.

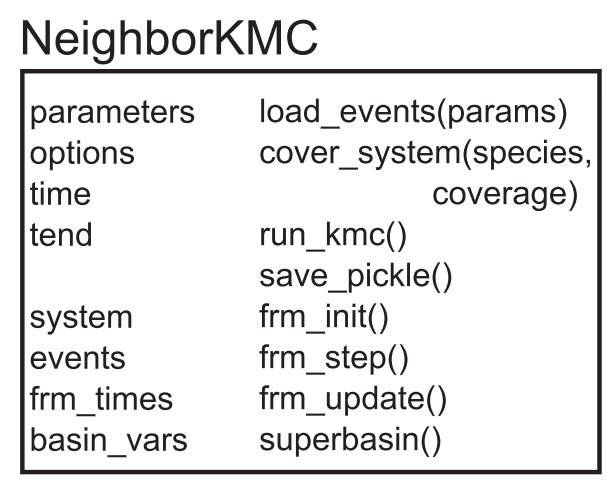

\section{Event}

\begin{tabular}{|c|c|}
\hline \multicolumn{2}{|c|}{$\begin{array}{l}\text { params, rev, alpha } \\
\text { possible(system, i_site, i_other) } \\
\text { get_rate(system, i_site, i_other) } \\
\text { do_event(system, i_site, i_other) }\end{array}$} \\
\hline System & Site \\
\hline $\begin{array}{l}\text { sites } \\
\text { atoms } \\
\text { neighbors } \\
\text { identify_neighbors() } \\
\text { get_ncovs(i_site) }\end{array}$ & $\begin{array}{l}\text { stype } \\
\text { covered } \\
\text { ind } \\
\text { neighbors }\end{array}$ \\
\hline
\end{tabular}

FIG. 3. The main classes, their variables, and functions.

The functions of the NeighborKMC class are mainly used to perform the simulation. Upon instantiating a NeighborKMC object, load_events (params) is called to populate the list events with the specified parameters such as temperature and pressures. params is a subset of parameters as discussed below. When instantiating a NeighborKMC object, cover_system (species, coverage) is also called to provide a certain coverage of surface species. run_kmc () sets time equal to zero and runs the kMC algorithm until time has reached tend. The function frm_init() initializes the $\mathrm{kMC}$ algorithm by generating the initial times of occurrences for the events. frm_step () takes a Monte Carlo step by performing the next possible event in the queue. After each step, frm_update () is called, which searches for events that have been enabled or disabled as a consequence of the performed step. superbasin() is responsible for handling the generalized temporal acceleration scheme by slowing down the quasi-equilibrated events during a simulation.

\section{B. Event class}

A $\mathrm{kMC}$ simulation proceeds using random numbers to perform different reactions and here these events are defined using classes. An Event class has three variables. A list of user-defined parameters (params) used to calculate the rate-constants. Examples of relevant parameters are pressures, temperatures, and molecular masses. params differs from the parameters variable of the NeighborKMC class as it only contains the relevant parameters for the Event class. The second variable is rev, which is an integer describing the index of the reverse reaction. The third variable is a lpha, 
which is the multiplication factor used to slow down quasiequilibrated events in the generalized temporal acceleration scheme.

The class contains three functions: One is a function possible (system, i_site, i_other) that during a simulation determines if the event can occur at site number i_site and co-participating sites indices i_other. The next function get_rate (system, i_site, i_other) is a user-defined function that, during simulation, returns the rate-constant for the given event on the specified sites. Finally, do_event (system, i_site, i_other) performs the event by modifying the relevant site objects in the system.

The user defines the types of reactive events by defining the event-types from the template base-class (EventBase) using inheritance. An example is to define COAdsEvent (EventBase) as a class that handles $\mathrm{CO}$ adsorption events. The parenthesis (EventBase) means that COAdsEvent is a class derived from EventBase. In this example, the possible (system, i_site, i_other) function returns True if the site is empty, and the get_rate (system, i_site, i_other) returns a rate-constant from collision theory. do_event (system, i_site, i_other) changes the site occupation of i_site to be occupied by a CO molecule.

Reaction energies used to calculate the rate-constants are stored in python dictionaries, which are connected to each userdefined event class. It is left up to the user to define how the rate-constants and energies are calculated and also to ensure thermodynamic consistency. The energies can be functions of occupations and alternative custom properties such as coordination numbers or site-types. The entropy changes during reactions and partition functions are also conveniently stored as member variables of the event-classes.

\section{System and site classes}

The System class contains three variables: A list of Site objects (sites), an optional ase.Atoms object (atoms), and a neighbor list to keep track of site-connectivity (neighbors). The sites list is used during the simulation to manipulate site-properties, such as coverage. atoms is used to associate ASE atoms with the kMC simulation, which can be used for visualization of the $\mathrm{kMC}$ run. The function identify_neighbors ( ) identifies neighboringsites that are connected and assigns them to the Site objects. This function is user-defined and it is typically based on distances between atoms in the atoms object. neighbors is the global neighbor-list consisting of the individual neighbor-lists of the sites. Thus, neighbors defines the global connectivity pattern. get_ncovs (i_site) returns the coverage of the nearest neighbors to the site with index i_site.

The Site class defines a site by the following standard variables: stype is a user-defined integer describing the type of site, which is used to distinguish the types of sites, such as edges and corners. If desired, additional variables can be added to describe the reaction energy landscape. For example, each site can be given a coordination number as a reaction energy descriptor and a strain to perturb the reaction energies. covered is an integer variable that describes the occupation of the site. The meaning of this integer is decided by the user, where, for example, covered $=0$ can represent empty sites and covered $=1$ a CO-covered site. Multidentate species can be implemented by changing the covered integer to a list covered $=$ [other_site_index, species_integer]. ind is a list of integers used to associate the site with an ase.Atoms object for visualization purposes. neighbors is a list of integers that contains the site index of neighbors-sites connected to the site. The indices in neighbors are not related to ind, but are instead associated with the global site list of the system object.

\section{Simulation setup}

To set up a simulation, the user needs to perform two initial steps. Step 1: Define the list of sites that defines the system. When defining the system, the function identify_neighbors () must be implemented. For example, this is achieved using the nearest neighbor distance between surface atoms in an ase. Atoms object. Step 2: Define one derived event-class for each possible elementary step in the reaction scheme. The specific event-objects are instantiated automatically at run time, and only the classes must be defined by the user prior to the simulation. In this step, reaction energies, entropic barriers, and rate-constants are defined.

\section{EXAMPLES}

The program has been used in different applications, ${ }^{20-22}$ and the following two examples are included to describe the workflow to set up a kMC simulation with MonteCoffee. Reaction energies in these examples are obtained by Density Functional Theory ${ }^{23,24}$ (DFT) calculations, with details presented in the Appendix.

\section{A. CO-oxidation on nanoparticles}

CO-oxidation over Pt nanoparticles is modeled by the following reaction scheme:

$$
\begin{aligned}
& \mathrm{CO}(\mathrm{g})+* \leftrightarrow \mathrm{CO}^{*}, \\
& \mathrm{CO}^{*}+* \leftrightarrow *+\mathrm{CO}^{*} \text { (Diffusion), } \\
& \mathrm{O}_{2}(\mathrm{~g})+2 * \leftrightarrow 2 \mathrm{O}^{*}, \\
& \mathrm{O}^{*}+* \leftrightarrow *+\mathrm{O}^{*} \text { (Diffusion), } \\
& \mathrm{CO}^{*}+\mathrm{O}^{*} \rightarrow \mathrm{CO}_{2}(\mathrm{~g})+2 *,
\end{aligned}
$$

where $*$ denotes an empty site. In this example, the sites are conveniently defined using an ase. Atom object. First an octahedral cluster is instantiated as an ase. Atom object by calling the following:

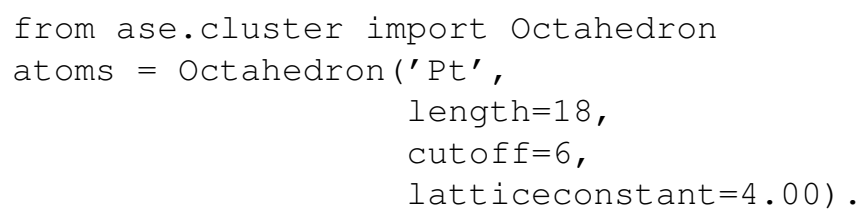

This instantiates an octahedral Pt nanoparticle with a lattice constant of $4 \AA$. The atoms object can be understood as a list 
containing ase. At om objects with information about chemical symbols, positions, charges, etc. Further details can be found in the ASE documentation. ${ }^{25}$

The generalized coordination number ${ }^{26,27}(\overline{\mathrm{CN}})$ of the sites is used to distinguish the types of sites. The $\overline{\mathrm{CN}}$ of a site is an extension of the conventional coordination number that is defined by the first nearest neighbor coordination. One site is defined for each surface atom on the particle, which is identified by having a conventional coordination number $<12$. The site in this example is a coarse-grained entity that entails ontop, bridge, and hollow positions. Figure 4 shows the $\overline{\mathrm{CN}}$ of ontop sites on the considered truncated octahedron by atomic coloring. Sites are instantiated with $50 \%$ randomly distributed $\mathrm{CO}$ coverage and zero oxygen coverage, which is set by the user in the cover_system() function. The system object is simply defined by the collection of sites and the function identify_neigbors (). Here, identify_neighbors () creates a neighbor list for each site based on distances in the associated ase. At oms object. Sites are neighbors if the associated atoms are separated by the fcc nearest-neighbor distance $\left(\frac{a}{\sqrt{2}}\right)$.

Reaction energies are based on DFT calculations using $\overline{\mathrm{CN}}$ as a descriptor (see the Appendix) as in Ref. 20. Adsorption energies, excluding adsorbate-adsorbate interactions, are stored as functions of $\overline{\mathrm{CN}}$ as

$$
\begin{aligned}
& E_{\mathrm{CO}, \mathrm{Pt}}^{\mathrm{ads}}(\overline{\mathrm{CN}})=1.403-0.252(\overline{\mathrm{CN}}-7.5), \\
& E_{\mathrm{O}, \mathrm{Pt}}^{\mathrm{ads}}(\overline{\mathrm{CN}})=0.975-0.218(\overline{\mathrm{CN}}-7.5) .
\end{aligned}
$$

The energy barrier for $\mathrm{CO}_{2}$ formation is calculated during the simulation through a Brønsted-Evans-Polanyi (BEP) relation in the $\mathrm{CO}$ and $\mathrm{O}$ adsorption energies

$$
E_{a}=2.95 \mathrm{eV}-0.824 \mathrm{eV} \times\left(E_{\mathrm{O}, \mathrm{Pt}}^{\mathrm{ads}}+E_{\mathrm{CO}, \mathrm{Pt}}^{\mathrm{ads}}\right) .
$$

Adsorbate-adsorbate interactions are based on the nearest neighbor occupations, which are retrieved as: system. get_ neighbor_covs (i_site). The adsorbate-adsorbate interactions are stored in a $3 \times 3$ python-list

$$
R=\left(\begin{array}{ccc}
0 & 0 & 0 \\
0 & E_{\mathrm{CO}-\mathrm{CO}} & E_{\mathrm{O}-\mathrm{CO}} \\
0 & E_{\mathrm{CO}-\mathrm{O}} & E_{\mathrm{O}-\mathrm{O}}
\end{array}\right)=\left(\begin{array}{ccc}
0 & 0 & 0 \\
0 & 0.19 & 0.30 \\
0 & 0.30 & 0.32
\end{array}\right) \mathrm{eV}
$$

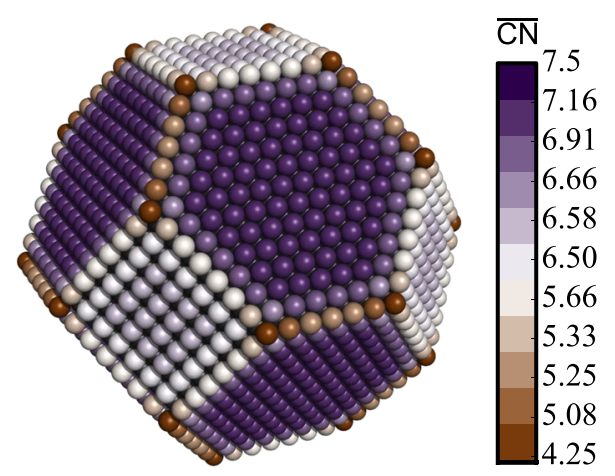

FIG. 4. Ontop sites on the truncated octahedron colored according to $\overline{\mathrm{CN}}$.
Thus, the repulsive energy addition $\delta E$ to species $1(\mathrm{CO})$ on a site is

$$
\delta E=\sum_{n} R_{1, c_{n}}
$$

Here $c_{n}$ is the occupation on the nearest neighbor $n$, which is 0 for empty sites, 1 for $\mathrm{CO}$, and 2 for $\mathrm{O}$. The repulsions are added to (4) and (5) during the simulation, which also affects the barrier through (6). Diffusion barriers between isoenergetic sites are set to $0.08 \mathrm{eV}$ for $\mathrm{CO}$ and $0.58 \mathrm{eV}$ for $\mathrm{O}$. These barriers are added to the difference in adsorption energies between the sites for thermodynamic consistency. To speed up simulation convergence, all diffusion barriers of $\mathrm{CO}$ are raised to a constant value of $0.45 \mathrm{eV}$. This value ensures convergence of the TOF. ${ }^{20}$ Similar approaches have been applied and justified previously. ${ }^{15-18,20,28,29}$

For each reactive event, one needs to define the function possible (system, i_site, i_other) that returns True if the event presently is possible, the function get_rate (system, i_site, i_other) that returns the rate constant, and the function do_event (system, i_site, i_other) that modifies the occupation according to the reaction. To enhance readability, we will not repeat the input arguments to these functions in the following discussion.

In the present example, one event is defined for each possible reaction in the scheme (3). For the $\mathrm{CO}$ adsorption event, a class named COAdsorption is derived from the base class EventBase. The class needs a number of parameters for calculating the rate-constants: temperature $(T)$, a constant $\mathrm{CO}$ pressure $\left(p_{\mathrm{CO}}\right), \mathrm{CO}$ mass $\left(m_{\mathrm{CO}}\right)$, the site area $\left(A_{\text {site }}\right)$, and the $\mathrm{CO}$ sticking coefficient $\left(s_{0}\right)$. These parameters are stored on the COAdsorption object. The possible () function returns True if the site is empty. The get_rate () function returns the following collision theory rate-constant:

$$
k_{i}^{\text {ads }}=\frac{A_{\text {site }} s_{0, i} p_{\mathrm{CO}}}{\sqrt{2 \pi m_{\mathrm{CO}} k_{\mathrm{B}} T}},
$$

where $i$ labels the site. The sticking coefficient of CO was set to 0.9 for the facet sites and to 1 for edges and corners.

The function do_event ( ) covers the site with species number $1(\mathrm{CO})$. This is done by invoking the following:

system.sites[site_index]. covered $=1$.

The oxygen adsorption event class is defined in complete analogy to CO adsorption. The possible() function returns True if the two neighboring sites passed to the function are unoccupied. The do_event () function changes the occupation of these sites to 2 (O covered).

To implement the desorption events, similar classes are defined. However, the desorption rate-constants are calculated from the equilibrium constants and adsorption rate-constants. The possible () function returns true if the site is covered with $1(\mathrm{CO})$ for $\mathrm{CO}$ desorption and two neighboring sites with 2 (O) for oxygen desorption. For CO desorption, get_rate ( ) returns the following desorption rate-constant:

$$
k_{i}^{\text {des }}=\frac{k_{i}^{\text {ads }}}{K_{i}}, \quad K_{i}=\exp \left(\frac{-\Delta G_{i}}{k_{\mathrm{B}} T}\right),
$$


where $\Delta G_{i}$ is the Gibbs free energy of adsorption, which depends on the adsorbate-adsorbate interactions. The enthalpic part of $\Delta G_{i}$ is given by (4) and (5). The entropic part of $\Delta G_{i}$ is implemented as the entropy difference between the gas-phase molecule in the ideal-gas approximation and the adsorbate in the harmonic approximation.

The $\mathrm{CO}_{2}$ formation event is modeled as irreversible. The possible () function returns true if $\mathrm{CO}$ is on the site and $\mathrm{O}$ is on the neighbor site passed to the function. get_rate () returns a rate-constant derived from transition state theory, ${ }^{30}$

$$
k_{i, j}^{\mathrm{TST}}=\frac{k_{\mathrm{B}} T}{h} \exp \left(\frac{-\Delta G_{i, j}^{\ddagger}}{k_{\mathrm{B}} T}\right),
$$

where $\Delta G_{i, j}^{*}$ is the Gibbs-free energy barrier for the $\mathrm{CO}^{*}+\mathrm{O}^{*}$ $\rightarrow \mathrm{CO}_{2}(\mathrm{~g})+2 *$ reaction with $\mathrm{CO}$ on site $i$ and $\mathrm{O}$ on site $j$. The enthalpy is defined in (6), and the entropic part of the barrier is treated in the harmonic approximation. do_event () simply assigns 0 to both the site and neighbor site in question.

Events are also defined for diffusion of adsorbed $\mathrm{CO}$ and O. possible () returns True if an adsorbate covers the site in question and the neighbor site is free. The get_rate () function returns a transition state theory rate equivalent to (11).

Figures 5(a) and 5(b) show the $\mathrm{CO}$ and $\mathrm{O}$ coverage on the different sites as a function of time at $700 \mathrm{~K}$. For $\mathrm{CO}$, the edges and corners have the highest coverages close to $100 \%$, whereas the coverages on the (100) and (111) facets are about $33 \%$ and $15 \%$, respectively. For oxygen, (100) has the highest coverage of ca $20 \%$, the (111) facets have about $10 \%$, whereas edges and corners have coverages below $5 \%$. The relative magnitudes of coverages are explained by the fact that corners have the highest reactivity, edges have the next highest reactivity, (100) and finally (111) sites have the lowest reactivity. The fact that edges and corners have small $\mathrm{O}$ coverages is owing to $\mathrm{CO}$ blocking. However, small fluctuations allow transient oxygen coverages on edges and corners. For both $\mathrm{CO}$ and $\mathrm{O}$, there is an initial equilibration period, which in the present case corresponds to roughly half the simulation time. The $\mathrm{CO}$ coverage has a larger fluctuation amplitude and frequency as compared to oxygen. Figure 5(c) shows the simulated Turnover frequency (TOF) as a function of temperature. The TOF is calculated by logging the number of times the $\mathrm{CO}_{2}$ formation event is performed after the initial equilibration. Error-bars stem from an average taken over 16 simulations. The TOF follows a light-off behavior where the value increases rapidly at about $800 \mathrm{~K}$. The rapid increase is due to $\mathrm{CO}$ desorption, which enables dissociative oxygen adsorption and thus $\mathrm{CO}_{2}$ formation. The present example illustrates how simulations over complex geometries can be performed by using an ase. Atoms object to define the sites and scaling relations to define the reaction energy landscape.

\section{B. Methane adsorption in zeolites}

To demonstrate the generality of the present implementation, in this example, methane adsorption is modeled in a zeolite. The adsorption, desorption, and diffusion are modeled using the following scheme:
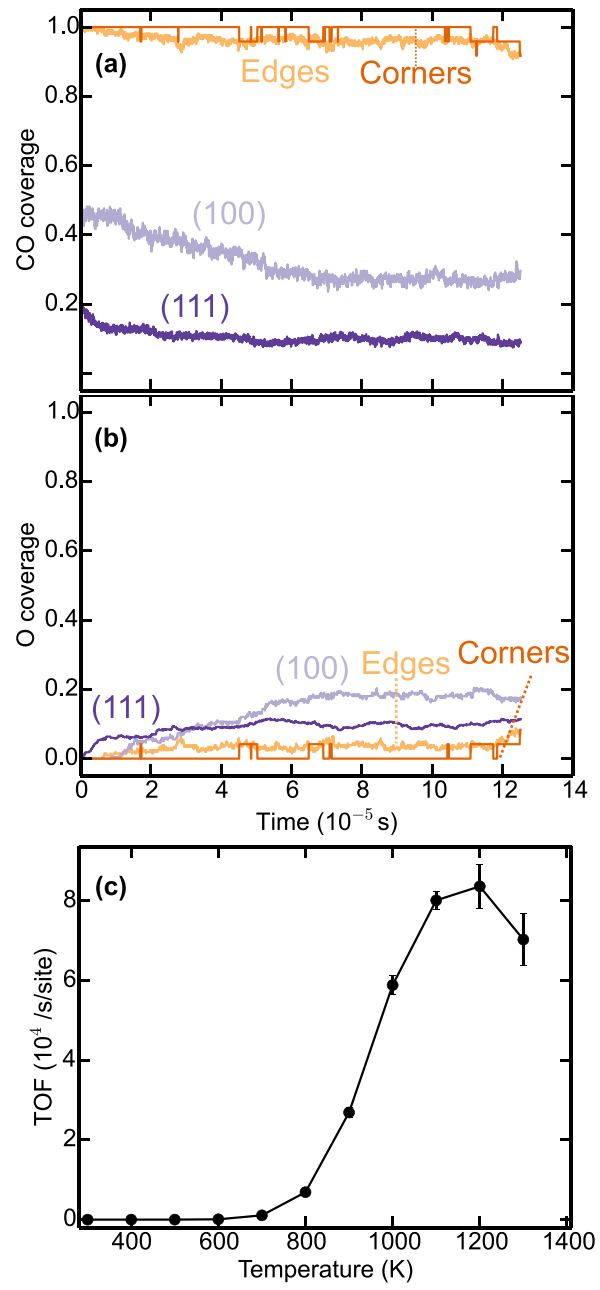

FIG. 5. (a) CO coverage and (b) O coverage of different site-types plotted as a function of time at $700 \mathrm{~K}$. (c) Average turnover frequency of $\mathrm{CO}$ oxidation as a function of temperature. $\mathrm{CO}$ pressure: $2 \mathrm{mbar}, \mathrm{O}_{2}$ pressure: 1 mbar.

$$
\begin{aligned}
\mathrm{CH}_{4}(\mathrm{~g})+* & \leftrightarrow \mathrm{CH}_{4}^{*}, \\
\mathrm{CH}_{4}^{*}+* & \leftrightarrow *+\mathrm{CH}_{4}^{*} .
\end{aligned}
$$

To simulate adsorption isotherms in a porous system such as a zeolite, the sites are conveniently put on a 3-dimensional grid of unit-cells. Such a grid is illustrated in Fig. 6, where there are four distinct types of cells (stype): 0-bulk, 1-facet, 2 -edge, and 3-corner. The facets have one entry available for adsorption, the edges have two, and the corners have three entries. The Site object is assigned a position vector $(i, j, k)$, which is used to identify the site-type. For example, for bulk sites, all indices are larger than zero and smaller than the number of sites-1. Non-bulk sites are exposed to the gas and are tagged with a custom variable gas_site $=$ True. The identify_neighbors() function identifies neighbor cells as those with distance 1 , which is calculated in the lattice-space by the metric

$$
d=\sqrt{\left(i_{2}-i_{1}\right)^{2}+\left(j_{2}-j_{1}\right)^{2}+\left(k_{2}-k_{1}\right)^{2}},
$$

where $d$ is the distance between two cells at positions $\left(i_{2}, j_{2}\right.$, $\left.k_{2}\right)$ and $\left(i_{1}, j_{1}, k_{1}\right)$.

The reactive events are defined in the next step. For $\mathrm{CH}_{4}$ adsorption, possible() returns True in empty non-bulk 


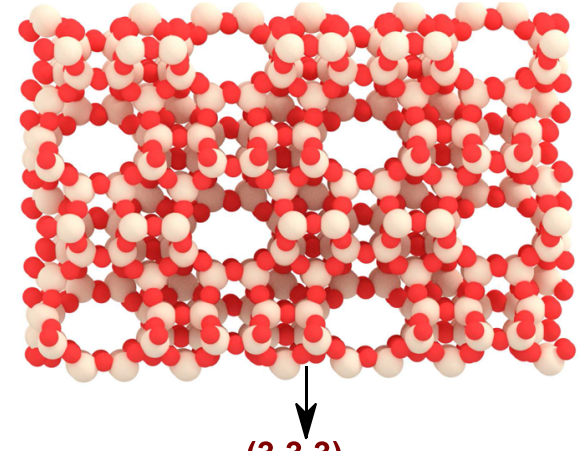

$(3,3,3)$

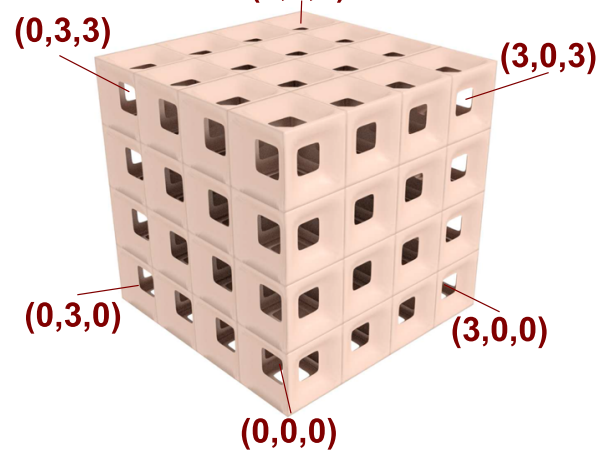

FIG. 6. Upper: Atomistic model of MFI zeolite channels. Lower: A cubic lattice model system with interconnected cells. Points indicate position in the lattice space.

cells (gas_site $=$ True). get_rate $($ ) returns the collision theory rate-constant with constant $\mathrm{CH}_{4}$ pressure as in (9), multiplied by the number of entries. do_event () simply changes system.sites[i_site].covered to 1 . For $\mathrm{CH}_{4}$ desorption, possible () returns True if the site is $\mathrm{CH}_{4}$ covered and non-bulk. The get_rate () function returns the desorption rate-constant defined in (10) multiplied by the number of entries, and do_event () changes covered to 0 .

The energy landscape is modeled by DFT calculations (see the Appendix). The $\mathrm{CH}_{4}$ adsorption energy in silicalite of zeolite framework type MFI silicalite is found to be $-0.38 \mathrm{eV}$, excluding zero point energy corrections. Barriers for $\mathrm{CH}_{4}$ diffusion between cells are arbitrarily set to $0.35 \mathrm{eV}$. The entropy of $\mathrm{CH}_{4}$ in the gas-phase is modeled in the ideal gas approximation and inside the framework it is approximated to be $2 / 3$ of the gas-phase entropy. ${ }^{31}$ The simulations are compared with the Langmuir model for the concentration,

$$
\theta_{\mathrm{LM}}=\frac{K}{1+K}, \quad K=\exp \left(\frac{-\Delta G}{k_{\mathrm{B}} T}\right),
$$

where $\Delta G$ is the Gibbs free energy change upon entering the zeolite from the gas-phase.

Figure 7 shows the simulated concentrations and the Langmuir result (14) as a function of temperature. At low temperatures, the predicted concentration is high and falls off as the temperature is increased. The concentrations predicted by the Langmuir model and the single unit-cell Monte Carlo are identical. However, the $20 \times 20 \times 20$ system gives slightly higher concentrations. This we attribute to pore-blocking effects where methane has to find a free route out from the bulk, which has been discussed previously in the context of zeolite deactivation. ${ }^{32}$ In reality, the pore-blocking effect is

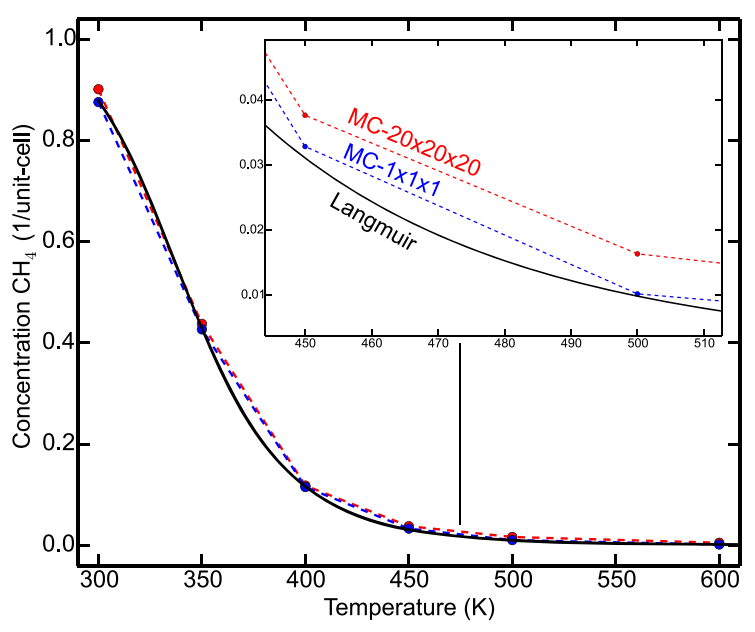

FIG. 7. Simulated methane concentration versus temperature at 1 mbar methane pressure: Langmuir model (black), Monte Carlo for $20 \times 20 \times 20$ unit-cells (red), and Monte Carlo for $1 \times 1 \times 1$ unit-cell (blue).

likely not pronounced for methane adsorption in MFI as two adsorbates could be present in the same cell. However, the present method is directly applicable to study pore-blocking phenomena in other systems. A conceptual difference between the kMC and the Langmuir model is configurational entropy. The kMC simulations include configurational entropy explicitly, whereas the Langmuir model is a mean-field theory that implements configurational entropy by assuming a random adsorbate distribution.

The present example illustrates that the object-oriented approach of MonteCoffee straightforwardly can simulate kinetics in a complex 3-dimensional pore network, such as a zeolite.

\section{CONCLUSION}

The MonteCoffee simulation framework enables rapid development of customized kinetic Monte Carlo (kMC) simulations, which is highly suitable for simulations that require complex geometries. The code is a programmable application written in python, which provides a basic framework for performing kMC simulations. Here we presented the structure and outline of the code. $\mathrm{CO}$ oxidation over Pt nanoparticles with a descriptor-based energy landscape and $\mathrm{CH}_{4}$ adsorption and diffusion inside zeolite pores were discussed as representative examples. MonteCoffee enables kMC simulations with full control over execution and rapid code development and thus it has the potential to considerably aid in the understanding of catalytic reaction kinetics.

\section{ACKNOWLEDGMENTS}

Financial support is acknowledged from Chalmers Excellence Initiative Nanoscience and Nanotechnology and the Swedish Research Council (2016-05234). The calculations were performed at PDC (Stockholm) and C3SE (Göteborg) via a SNIC grant. The Competence Centre for Catalysis (KCK) is hosted by Chalmers University of Technology and is financially supported by the Swedish Energy Agency and the member companies AB Volvo, ECAPS AB, Johnson Matthey AB, 
Preem AB, Scania CV AB, Umicore Denmark ApS, and Volvo Car Corporation AB.

The modules are available at https://gitlab.com/ ChemPhysChalmers/MonteCoffee.

\section{APPENDIX: DENSITY FUNCTIONAL THEORY}

Reaction energies were calculated by density functional theory with the Vienna $a b$ initio simulation (VASP) package. ${ }^{33-35}$ A plane-wave basis with a kinetic cutoff of $450 \mathrm{eV}$ was used. Core-valence electronic interactions were treated in the Projector-Augmented Wave (PAW) scheme with valence electrons: $\mathrm{Pt}(10), \mathrm{Si}(4), \mathrm{O}(6), \mathrm{C}(4)$, and $\mathrm{H}(1)$. Ionic relaxations were performed in ASE with the BFGS-Linesearch algorithm using a force convergence criterion of $0.05 \mathrm{eV} / \AA$. Gas-phase molecules were relaxed in cells of at least $(22 \AA \times 22 \AA$ $\times 22 \AA$ ). Vibrational energies were calculated in the harmonic approximation with finite differences of displacement $0.01 \AA$. Spin-polarization was applied only to $\mathrm{O}_{2}$ in the gas-phase, which was treated in the triplet spin-state. Energy barriers were evaluated using the Nudged Elastic Band ${ }^{36}$ (NEB) function from the Variational Transition State Theory (VTST) tools ${ }^{37}$ with seven images. Initial interpolations between the initial and final state were done using the image dependent pair potential. ${ }^{38}$

The reaction energies for $\mathrm{CO}$ oxidation over nanoparticles were calculated in Ref. 20 using the Revised PerdewBurke-Ernzerhof (RPBE) ${ }^{39}$ exchange-correlation functional. The Pt lattice-constant was calculated in the fcc unit cell to be $4.00 \AA$ using a $(12 \times 12 \times 12) \mathrm{k}$-point grid. Model surfaces were treated as four-layer slabs in at least $(2 \times 2)$ cells using $(6 \times 6 \times 1)$ k-points. A $12 \AA$ vacuum was introduced perpendicular to the slab separating periodic images.

In the zeolite-example, the Bayesian Error Estimation Functional with van der Waals correlation (BEEF-vdW) ${ }^{40}$ was used to model exchange and correlation. The adsorption energy of $\mathrm{CH}_{4}$ was found by local relaxation initiated from multiple positions of the molecule.

${ }^{1}$ J. K. Nørskov, T. Bligaard, J. Rossmeisl, and C. H. Christensen, Nat. Chem. 1, 37 (2009).

${ }^{2}$ M. J. Hoffmann, M. Scheffler, and K. Reuter, ACS Catal. 5, 1199 (2015).

${ }^{3}$ M. Stamatakis and D. G. Vlachos, ACS Catal. 2, 2648 (2012).

${ }^{4}$ D. T. Gillespie, J. Comput. Phys. 22, 403 (1976).

${ }^{5}$ K. A. Fichthorn and W. H. Weinberg, J. Chem. Phys. 95, 1090 (1991).

${ }^{6}$ M. J. Hoffmann, S. Matera, and K. Reuter, Comput. Phys. Commun. 185, 2138 (2014).
${ }^{7}$ J. J. Lukkien, "Carlos," accessed online 24 January 2018, https://carlos. win.tue.n1/.

${ }^{8}$ S. S. Plimpton, A. Thompson, and A. Slepoy, "Spparks kinetic monte carlo simulator," accessed online 24 January 2018, http://spparks.sandia.gov/.

${ }^{9}$ M. Leetmaa and N. V. Skorodumova, Comput. Phys. Commun. 185, 2340 (2014).

${ }^{10}$ M. Leetmaa and N. V. Skorodumova, Comput. Phys. Commun. 196, 611 (2015).

${ }^{11}$ F. M. Kunz, L. Kuhn, and O. Deutschmann, J. Chem. Phys. 143, 044108 (2015).

${ }^{12}$ M. Stamatakis and D. G. Vlachos, J. Chem. Phys. 134, 214115 (2011).

${ }^{13}$ P. F. Dubois, Comput. Phys. 8, 70 (1994).

${ }^{14}$ S. R. Bahn and K. W. Jacobsen, Comput. Sci. Eng. 4, 56 (2002).

${ }^{15}$ A. P. J. Jansen, An Introduction to Kinetic Monte Carlo Simulations of Surface Reactions (Springer, Berlin, 2012), pp. 11-12; 38; 53; 162-163.

${ }^{16}$ A. Chatterjee and A. F. Voter, J. Chem. Phys. 132, 194101 (2010).

${ }^{17}$ M. Stamatakis and D. G. Vlachos, Comput. Chem. Eng. 35, 2602 (2011).

${ }^{18}$ E. C. Dybeck, C. P. Plaisance, and M. Neurock, J. Chem. Theory Comput. 13, 1525 (2017).

${ }^{19}$ See https://docs.python.org/2/tutorial/classes.html for a description of object oriented programming in python; accessed online 15 May 2018.

${ }^{20}$ M. Jørgensen and H. Grönbeck, ACS Catal. 7, 5054 (2017).

${ }^{21}$ M. Jørgensen and H. Grönbeck, Angew. Chem., Int. Ed. 57, 5086 (2018).

${ }^{22}$ T. Nilsson Pingel, M. Jørgensen, A. Yankovich, H. Grönbeck, and E. Olsson, Nat. Commun. 9, 2722 (2018).

${ }^{23}$ P. Hohenberg and W. Kohn, Phys. Rev. 136, B864 (1964).

${ }^{24}$ W. Kohn and L. J. Sham, Phys. Rev. 140, A1133 (1965).

${ }^{25} \mathrm{See} \mathrm{https://wiki.fysik.dtu.dk/ase/ase/atoms.html} \mathrm{for} \mathrm{a} \mathrm{description} \mathrm{of} \mathrm{the} \mathrm{ase}$ atoms object; accessed online 15 June 2018.

${ }^{26}$ F. Calle-Vallejo, J. I. Martínez, J. M. García-Lastra, P. Sautet, and D. Loffreda, Angew. Chem., Int. Ed. 53, 8316 (2014).

${ }^{27}$ F. Calle-Vallejo, D. Loffreda, M. T. M. Koper, and P. Sautet, Nat. Chem. 7, 403 (2015).

${ }^{28}$ B. Puchala, M. L. Falk, and K. Garikipati, J. Chem. Phys. 132, 134104 (2010).

${ }^{29}$ K. A. Fichthorn and Y. Lin, J. Chem. Phys. 138, 164104 (2013).

${ }^{30}$ H. Eyring, Chem. Rev. 17, 65 (1935).

${ }^{31}$ C. Paolucci, A. A. Parekh, I. Khurana, J. R. D. Iorio, H. Li, J. D. Albarracin Caballero, A. J. Shih, T. Anggara, W. N. Delgass, J. T. Miller, F. H. Ribeiro, R. Gounder, and W. F. Schneider, J. Am. Chem. Soc. 138, 6028 (2016).

${ }^{32}$ D. Cai, Y. Ma, Y. Hou, Y. Cui, Z. Jia, and C. Zhang, Catal. Sci. Technol. 7, 2440 (2017).

${ }^{33}$ G. Kresse and J. Furthmuller, Phys. Rev. B 54, 11169 (1996).

${ }^{34} \mathrm{G}$. Kresse and J. Furthmuller, Comput. Mater. Sci. 6, 15 (1996).

${ }^{35} \mathrm{G}$. Kresse and J. Hafner, Phys. Rev. B 47, 558 (1993).

${ }^{36}$ G. Henkelman, B. P. Uberuaga, and H. Jónsson, J. Chem. Phys. 113, 9901 (2000).

${ }^{37} \mathrm{See}$ http://theory.cm.utexas.edu for "Henkelman Group at the University of Texas at Austin Home Page" (accessed November 16, 2015).

${ }^{38} \mathrm{~S}$. Smidstrup, A. Pedersen, K. Stokbro, and H. Jónsson, J. Chem. Phys. 140, 214106 (2014).

${ }^{39}$ B. Hammer, L. B. Hansen, and J. K. Nørskov, Phys. Rev. B 59, 7413 (1999).

${ }^{40} \mathrm{~J}$. Wellendorff, K. T. Lundgaard, A. Møgelhøj, V. Petzold, D. D. Landis, J. K. Nørskov, T. Bligaard, and K. W. Jacobsen, Phys. Rev. B 85, 235149 (2012). 